\title{
Robust Smoothness Estimation in Statistical Parametric Maps Using Standardized Residuals from the General Linear Model
}

\author{
Stefan J. Kiebel, * Jean-Baptiste Poline, $†$ Karl J. Friston, $\ddagger$ Andrew P. Holmes, $\ddagger$ and Keith J. Worsley ${ }^{\S}$ \\ *Department of Neurology, Friedrich-Schiller-University, Jena, Germany; †CEA Service Hospitalier Frédéric Joliot, Orsay, France; \\ $\ddagger$ The Wellcome Department of Cognitive Neurology, The Institute of Neurology, London, United Kingdom; \\ and ${ }^{\S}$ Department of Mathematics and Statistics, McGill University, Montreal, Québec, Canada
}

Received May 27, 1999

The assessment of significant activations in functional imaging using voxel-based methods often relies on results derived from the theory of Gaussian random fields. These results solve the multiple comparison problem and assume that the spatial correlation or smoothness of the data is known or can be estimated. End results (i.e., $P$ values associated with local maxima, clusters, or sets of clusters) critically depend on this assessment, which should be as exact and as reliable as possible. In some earlier implementations of statistical parametric mapping (SPM) (SPM94, SPM95) the smoothness was assessed on Gaussianized $t$-fields (Gt-f) that are not generally free of physiological signal. This technique has two limitations. First, the estimation is not stable (the variance of the estimator being far from negligible) and, second, physiological signal in the Gt-f will bias the estimation. In this paper, we describe an estimation method that overcomes these drawbacks. The new approach involves estimating the smoothness of standardized residual fields which approximates the smoothness of the component fields of the associated $t$-field. Knowing the smoothness of these component fields is important because it allows one to compute corrected $\boldsymbol{P}$ values for statistical fields other than the $t$-field or the Gt-f (e.g., the $F$-map) and eschews bias due to deviation from the null hypothesis. We validate the method on simulated data and demonstrate it using data from a functional MRI study. $\odot 1999$ Academic Press

\section{INTRODUCTION}

The objective of voxel-based analyses of functional brain images is to classify measured activities as significant on the basis of the probability that they could have occurred by chance. Estimation of these probabilities can be based on the theory of Gaussian random fields (GRF) (Adler, 1981), which is used to describe certain features of functional brain images and the associated statistic image process. It is important that the correlations of neighbouring voxels in statistical processes are taken into account when solving the multiple comparison problem and estimating the significance of an activation. To quantify these correlations, the smoothness of the statistical process must be estimated under the null hypothesis that the statistical process does not contain any signal due to physiological changes. The smoothness estimator in the framework of older implementations of statistical parametric mapping (SPM) (SPM94, SPM95) used the observed statistical process, which is generally not free of effects induced by the experimental paradigm. In this note, we describe a new method that uses mathematical results from Worsley (1996), which eschews this problem and estimates smoothness in an unbiased way. To validate these findings, we show that the new method accurately estimates the smoothness of simulated statistical processes. We apply the new estimator to a functional MRI study to exemplify the procedure and observe that the new smoothness estimator is more accurate than the previous one.

\section{THEORY}

\section{Preliminaries}

One of the aims of functional brain data analysis techniques is to find a solution for the multiple comparison problem, which is to characterize a computed univariate statistical map in such a way that the chance of occurrence of interesting events is estimated correctly under the null hypothesis that there are no effects induced by the experimental design. This task is not as simple as it first appears to be, since neighboring statistical voxel values are not independent because of the intrinsic point spread function of the imaging device, the autocorrelation in physiological effects, or smoothness induced by an exogenous spatial lowpass filter employed prior to analysis.

Modeling this knowledge about the underlying spatial autocorrelation of the signal or the smoothness leads quite naturally to methods which use some 
measure of autocorrelation to detect activation effects deviating from the expected spatial features within a given statistical map. The theory of Gaussian random fields (Adler, 1981) seems to be a framework appropriate for characterizing statistical maps and detecting spatial activation effects. Using the theory of random fields, the chance of occurrence of events (local voxel maxima, cluster sizes, or cardinality of a set of clusters) in a statistical process can be assessed under the assumption that the discrete process in question approximates a GRF that conforms to this characterization. Any event in the statistical image whose $P$ value for occurrence by chance in the GRF is sufficiently small can be declared significant. In the framework of SPM, the statistical process is a Gaussianized $t$-field (Gt-f), which is taken to be a good lattice representation of a stationary Gaussian random field for high degrees of freedom. (See, e.g., Holmes, 1994, for a review of the underlying assumptions.) The important properties of a stationary GRF are that (1) the distribution of any voxel value is normal with zero mean and unit variance, (2) any subset of voxel values has a multivariate normal distribution, and (3) this multivariate distribution is strictly stationary (i.e., is not a function of position). These properties imply that the point response function (PRF) of the Gt-f under the null hypothesis is stationary; i.e., the generating parameters of a stationary GRF are its mean, variance, and the autocorrelation function (ACF), where the parametric form of the ACF itself does not need to be Gaussian, but has properties (2) and (3) of a stationary GRF. Since the moments of the statistical process under the null hypothesis are known, the smoothness induced by the ACF (under the null hypothesis) remains to be assessed.

The smoothness of a stationary GRF is defined as $|\Lambda|^{-1 / 2}$, where $\Lambda$ is the covariance matrix of the partial derivatives of the GRF at any voxel position. This definition of smoothness is a function of the underlying (in most cases unknown) parametric form of the PRF. However, to estimate the unknown $\Lambda$, it is not necessary to estimate the actual PRF or the associated ACF. The only assumption made is that the ACF must be twice differentiable at zero. This reflects the fact that smoothness estimation is only concerned with the features of the ACF around zero.

If the underlying parametric form of the PRF is assumed to be Gaussian, another way of describing the smoothness is the covariance matrix $W=(2 \Lambda)^{-1}$ of the Gaussian filter, which could have been used to generate the smoothness of the GRF in question from a Gaussian white noise process.

If the off-diagonal elements of $W$ are zero, meaning that the PRF is elliptical in shape, with axes aligned with the coordinate axes, each image dimension has its own smoothness measure, independent of other dimen- sions. This sparse way of reporting smoothness is often used for a Gaussian PRF, for which we measure smoothness as full width at half maximum (FWHM) in each dimension: $\mathrm{FWHM}_{i}=\sqrt{8 \ln (2) W_{i i}}$ for $i=1, \ldots, D$.

\section{Inference}

In the following, we will summarize some of the important results concerning statistical inference in voxel-based functional brain imaging based on the theory of random fields.

Although the following equations look rather complex, it is important to note that only the smoothness parameter $|\Lambda|$ is unknown. Since it would be beyond the scope of this work to derive the equations, we refer the reader to the cited articles.

The first statistical inference method based on smoothness of the statistical process was described by Friston et al. (1991). In this work, a test for peak height for two-dimensional data was proposed. This method effectively solved the problem of mass, nonindependent testing. Worsley et al. (1992) proposed a test, which could be applied to data of any dimension $D$ using the expected Euler characteristic of a GRF. These methods were based on the probability that the maximum value $Z_{\text {max }}$ in a GRF is higher than a high threshold $t$,

$$
P\left(Z_{\max }>t\right) \approx V|\Lambda|^{1 / 2}(2 \pi)^{-(D+1) / 2} H e_{D}(t) e^{-t^{2} / 2},
$$

where $H e_{D}(t)$ is the Hermite polynomial of degree $D$ in $t$.

Worsley et al. (1992) assumed homoscedasticity (i.e., assumed the same error variance at each voxel) giving a Gaussian statistical image so that he could directly apply Eq. (1). When this assumption cannot be made, a $t$-field statistic must be computed, where the degrees of freedom used for variance estimation at each voxel is dependent on the rather low number of observations (scans) instead of the high number of voxels.

Friston et al. (1994), building on the results of Worsley et al. (1992), derived a formula for the probability that the size $n_{t}$ of a cluster in a GRF above a high threshold $t$ exceeds $k$ voxels,

$$
P\left(n_{t}>k\right)=1-\exp \left(-E(m) e^{-\beta k^{2} / D}\right),
$$

where $E(m) \approx P\left(Z_{\max }>t\right)$ is the expected number of clusters for threshold $t$ and

$$
\beta=\left(\frac{\Gamma(D / 2+1) E(m)}{V \Phi(-t)}\right)^{2 / D} .
$$

In Friston et al. (1996), a formula for the probability that a set of clusters $c$ in a GRF above threshold $t$ and size greater than $n$ has cardinality greater than or equal to $k$ was presented. Let $C_{n, t}$ be the cardinality of $c$ 


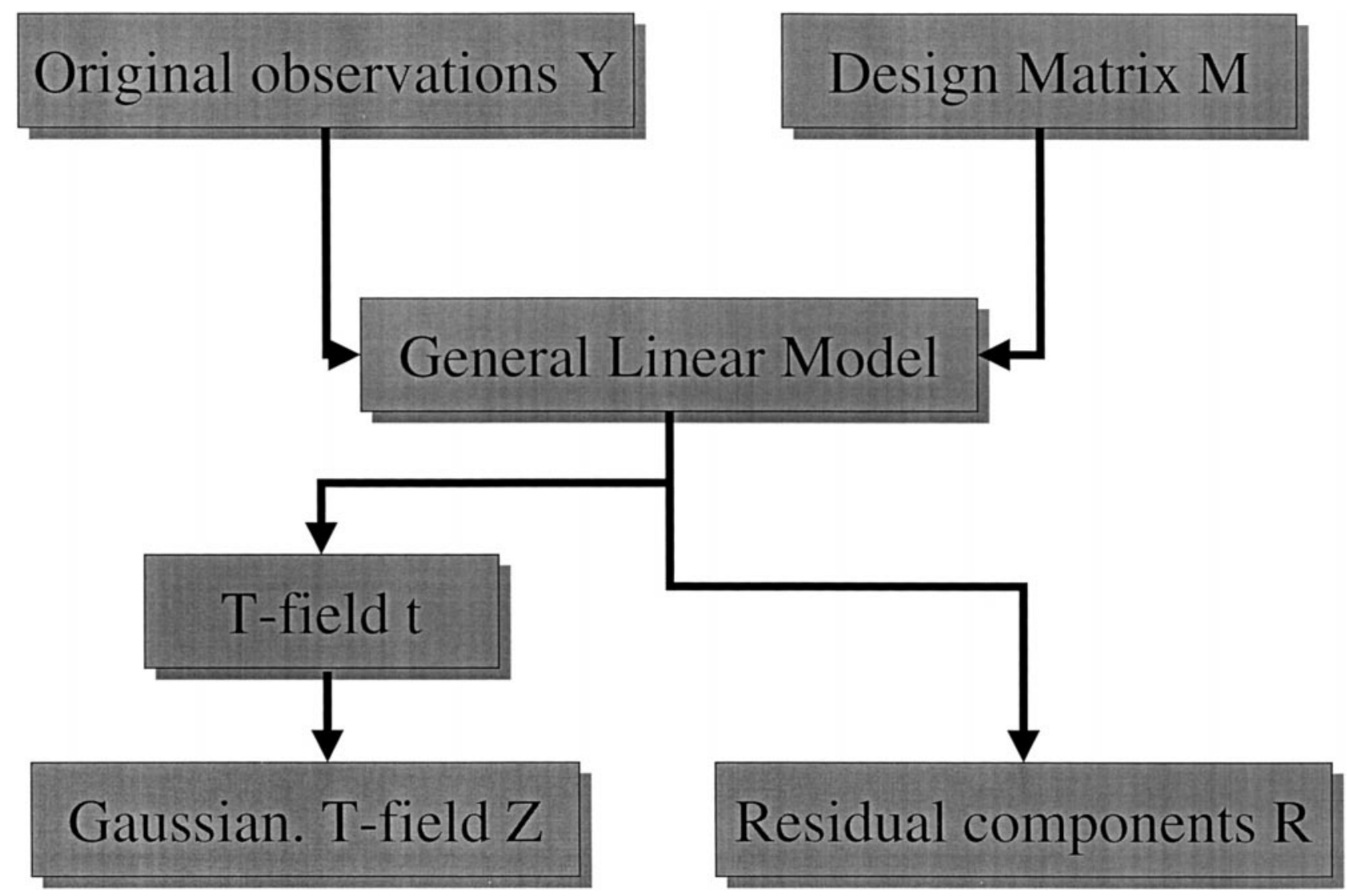

FIG. 1. Flow of information in SPM.

with cluster size greater $n$ and generating threshold $t$ in volume $V$. Then

$P\left(\left(C_{n, t} \geq k\right)=1-\sum_{i=0}^{k-1} \operatorname{poisson}\left(i, E(m) \cdot p\left(n_{t} \geq n\right)\right)\right.$,

where

$$
\text { poisson }(i, p)=\frac{e^{-p_{p} i}}{i !}
$$

Formulas for the test for peak height are not only known for Gaussian random fields (Eq. (1)), but also for $\chi^{2}-, F$-, and $t$-fields, whereas formulas for other tests (supratreshold cluster size and set level inference, Eqs. (2) and (3)) are only known for Gaussian random fields. This means that analysis methods assuming heteroscedasticity (e.g., SPM) must transform the resulting $t$-field to a Gaussianized $t$-field (see next section) to apply these results for low degrees of freedom.

As mentioned above, all variables in Eqs. (1)-(3) except for the smoothness are known or can be directly deduced from the observed data. Therefore $|\Lambda|$ remains the only parameter which must be estimated.

\section{Processing Concepts}

In what follows, we give a short summary of the general processing strategy used to analyze functional images in a univariate way. It is important to note that the specification of the underlying mathematical models is not only an abstract way to describe the univariate functional analysis as it is performed in SPM, but many other possible (and existing) methods could be described by these or very similar models.

The univariate method implemented in SPM uses the same linear model at each voxel position so that we can drop the voxel position subscript in the following. To aid the understanding of the formal description, Fig. 1 shows a flow chart of the information processing done in SPM. The general linear model at each voxel position is given by

$$
\mathbf{Y}=M \beta+\sigma \epsilon
$$

where $Y$ is the observed image intensity vector for images $i=1, \ldots, n, M$ is the $n \times p$-design matrix of the model, $\beta$ is a $p$-dimensional parameter vector, $\sigma$ is the (scalar) standard deviation of $\mathbf{Y}$, and $\epsilon$ is a vector of normal errors. 
To ensure robust parameter estimates in the case of temporally autocorrelated data (fMRI), the model is convolved with an approximate hemodynamic response function, giving us the extended general linear model

$$
K \mathbf{Y}=K M \beta+K \sigma \epsilon,
$$

where $K$ is a Toeplitz temporal smoothing matrix. The correlation matrix of $\epsilon$ is assumed to be a known matrix $V$. For PET data the scans are independent so $V=I_{n}$, the $n \times n$ identity matrix. For fMRI data the errors are assumed to be autocorrelated, but after temporal smoothing $V \sim K K^{\prime}$.

After estimating $\beta$ by the least-squares method, the residuals are given by

$$
\mathbf{R}=K(\mathbf{Y}-M \hat{\beta})
$$

and $R_{i}$ are the residual fields consisting of $R_{i}(\mathbf{l})$ for all voxel positions $\mathbf{1}$. Similarly, the $\epsilon_{i}$ are the (unobservable) error fields of the underlying $t$-field consisting of $\epsilon_{i}(\mathbf{l})$ for all voxel positions $\mathbf{l}$. In the following, we will refer to these $\epsilon_{i}$ as component fields of a $t$-field.

The residual forming projection matrix is given by

$$
\left.P=I_{n}-M^{*}\left(M^{* \prime} M^{*}\right)^{-1} M^{* \prime}\right),
$$

where $M^{*}=K M$.

The variance $\sigma^{2}$ (of the temporally convolved observations) is estimated by dividing the sum of squares of the residuals by its expectation, i.e.,

$$
\hat{\sigma}^{2}=\frac{\mathbf{R}^{\prime} \mathbf{R}}{\operatorname{trace}(P V)} .
$$

Then a $t$-field

$$
t=\frac{\mathbf{c}^{\prime} \hat{\beta}}{\left(\mathbf{c}^{\prime} \hat{\sigma}^{2}\left(M^{* \prime} M^{*}\right)^{-1} M^{* \prime} V M^{*}\left(M^{* \prime} M^{*}\right)^{-1} \mathbf{c}\right)^{1 / 2}} \sim t_{v}
$$

is computed to test the hypothesis that $\mathbf{c}^{\prime} \beta=0$, where $\mathbf{c}$ is a suitable contrast vector (Frackowiak et al., 1997) and

$$
v=\frac{\operatorname{trace}(P V)^{2}}{\operatorname{trace}(P V P V)}
$$

are the effective degrees of freedom (Worsley and Friston, 1995).

Finally, the $t$-field $t$ is Gaussianized to the Gt-f $Z=$ $f(t)$, where the transform function is given by

$$
f(t)=\Phi^{-1}\left(\Psi_{v}(t)\right),
$$

where $\Phi^{-1}$ is the inverse standard normal cumulative density function (CDF) and $\Psi_{v}$ is the CDF of the Student's $t$-distribution with $v$ degrees of freedom. This Gt-f $Z$ is assumed to approximate a continuous GRF with zero mean and unit variance under the null hypothesis for high degrees of freedom.

\section{Smoothness Estimation}

To apply the theory of Gaussian random fields and to evaluate Eqs. (1)-(3), the covariance matrix $\Lambda_{Z_{0}}$ of the partial derivatives of $Z_{0}$ must be estimated, where $Z_{0}$ denotes the Gt-f $Z$ under the null hypothesis.

Although it is not possible to directly observe $Z_{0}$ and estimate $\Lambda_{Z_{0}}$, a solution can be found based on the smoothness estimation of the component fields $\epsilon_{i}$ of the $t$-field. The overall strategy consists of two steps: First, estimate the covariance matrix $\Lambda_{\epsilon}=\Lambda_{\epsilon_{i}}$ for any $i$ of the partial derivatives of $\epsilon_{i}$, which can be assessed by an estimator based on the residual fields $R_{i}$ (Worsley, 1996). Second, a scalar correction factor suffices to relate $\hat{\Lambda}_{\epsilon}$ to $\hat{\Lambda}_{Z_{0}}$, thus giving us an estimator for the smoothness of $Z_{0}$. In current versions of SPM (e.g., SPM99) this second step is no longer necessary because inference is based directly on the $t$ - or $F$-map without the need for Gaussianization.

Figure 2 shows the relationships between the different fields and their smoothness estimates. We assume here that all $\epsilon_{i}$ have the same $\Lambda_{\epsilon}$ so that we can use all $\epsilon_{i}$ to estimate the resulting smoothness of the component fields. This assumption does not necessarily follow from our assumption about the strict stationarity of the PRF used to generate $Z_{0}$, since stationarity applies only to the PRF within a GRF and not to PRFs over different GRFs. However, for functional brain imaging data, the assumption of approximately equal PRFs over different component fields can be made, since the data are usually acquired from the same scanner under similar conditions and the same exogenous filter kernel is applied to the data prior to the statistical analysis. Additionally, smoothness (in the framework of SPM) is only measured within brain tissue locations.

In the following, we describe the estimation of $\Lambda_{\epsilon}$ based on the residuals $R_{i}$ and derivation of a scalar factor to relate $\hat{\Lambda}_{\epsilon}$ to $\hat{\Lambda}_{Z_{0}}$ (Worsley et al., 1992).

Estimating the smoothness of the component fields. The covariance matrix of the first partial derivatives of $\epsilon_{i}$, given by

$$
\Lambda_{\epsilon}=\operatorname{Var}\left(\frac{\partial \epsilon_{i}}{\partial \mathbf{x}}\right)
$$

for any $i=1, \ldots, n$ must be estimated. 


\section{Residual components $\boldsymbol{R}$}

\section{Normalised Residual}

components $S$

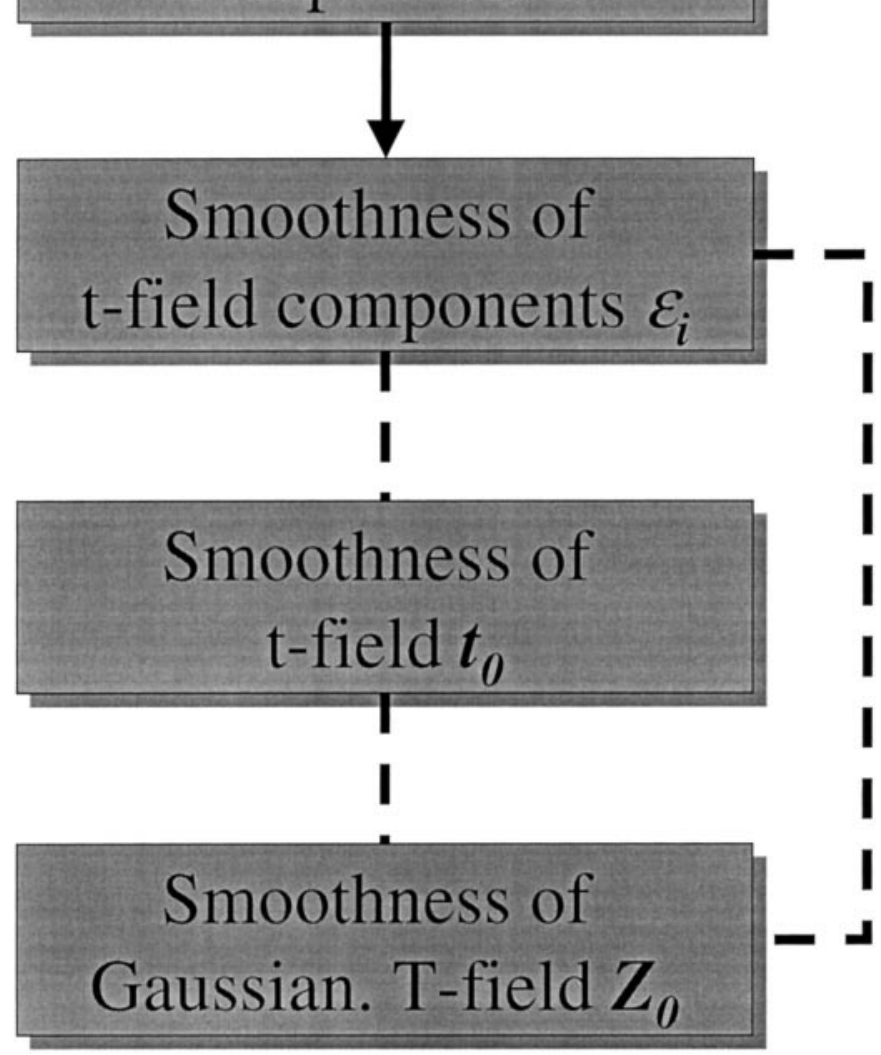

FIG. 2. Smoothness estimation of the null fields based on the residuals of the general linear model. The solid lines (with arrow) indicate computation of a field or estimation of smoothness based on a field, whereas the dashed lines stand for estimation of smoothness based on a scalar correction factor. The relations described by dashed lines are valid in both directions.

An estimate $\Lambda_{\epsilon}$ can be found based on the estimated $\epsilon_{i}$. We define the standardized residuals by

$$
\mathbf{S}=\hat{\mathbf{E}}=\frac{\mathbf{R}}{\sqrt{\mathbf{R}^{\prime} \mathbf{R}}}
$$

Worsley (1996) has shown that an unbiased estimator of $\lambda_{j k}$, the covariance of the partial derivatives of the components $\epsilon_{i}$ in direction $j$ and $k$, is given by

$$
\hat{\lambda}_{j k}=\frac{v-2}{(v-1) N} \sum_{\mathbf{x}} \sum_{i}^{n} \frac{\partial S_{i}}{\partial l_{j}} \frac{\partial S_{i}}{\partial l_{k}},
$$

where $N$ is the total number of all positions $\mathbf{x}$. In the discrete case, the numerical computation of the partial derivatives via the gradient operator is given by

$$
\nabla S_{j}=\frac{S_{j+1}-S_{j-1}}{2 \delta d}
$$

for nonedge voxels $x_{j}$ of a discrete field and step size $\delta d$.

Since the normalizing denominator of the $S_{i}$ is not the true voxel-level standard deviation, but an estimate with its own error, additional variance is introduced to the estimated component fields (compared to the true and unknown component fields). Thus the smoothness of the estimated component fields will be slightly lower, where the amount of underestimation is a function of the effective degrees of freedoms $v$. To yield an unbiased estimator (Eq. (14)), the factor $(v-2) / v-1)$ must be used. For a proof of unbiasedness, see Worsley (1996).

The covariances of the first partial derivatives of functional brain imaging processes can be assumed to be zero (Poline et al., 1995) so that the GRF examined can be generated by a separable filter kernel. Therefore, in the framework of SPM, only the variances of $\Lambda_{\epsilon}$ are estimated by

$$
\hat{\lambda}_{j j}=\frac{v-2}{(v-1) N} \sum_{\mathbf{x}} \sum_{i}^{n}\left(\frac{\partial S_{i}}{\partial l_{j}}\right)^{2} .
$$

Estimating the smoothness of $Z_{0}$. It remains to be shown that $\Lambda_{\epsilon}$ is directly related to $\Lambda_{Z_{0}}$. Under the null hypothesis and provided that the $\epsilon_{i}$ are discrete realizations of a stationary GRF, $Z_{0}$ is an approximation of a GRF. Though the PRF for the $\epsilon_{i}, t_{0}$ (the $t$-field under the null hypothesis), and $Z_{0}$ are not the same except for very high (infinite) degrees of freedom, these three sorts of fields are all dependent on the underlying PRF such that the smoothnesses of $t_{0}$ and the Gt-f $Z_{0}$ can be related to the smoothness of the $\epsilon_{i}$ as shown in Worsley et al. (1992).

Thus, the covariance matrix of the Gt-f $Z_{0}$ can be computed by

$$
\hat{\Lambda}_{Z_{0}}=\lambda_{v} \cdot \hat{\Lambda}_{\epsilon}
$$

where the correction factor

$$
\lambda_{v}=\int_{-\infty}^{\infty} \frac{\left(t^{2}+n-1\right)^{2}}{(v-1)(v-2)} \frac{T p d f_{v}(t)^{3}}{p(t)^{2}} d t,
$$


where $T p d f_{v}$ is the probability density function (PDF) of a $t$-distribution with $v$ degrees of freedom, $p(t)=$ $\phi\left(\Phi^{-1}\left(1-\Phi_{\nu}(t)\right)\right)$ and $\phi(z)$ is the PDF of the standard normal distribution. This integral does not converge for $v<3$ and approximates 1 for $v \rightarrow$ inf. As implicit in the derivation of $\lambda_{v}$, a similar simple relationship exists between $\Lambda_{\epsilon}$ to $\Lambda_{t_{0}}$ and between $\Lambda_{t_{0}}$ and $\Lambda_{Z_{0}}$.

A biased estimator. A much simpler way to estimate $\Lambda_{Z_{0}}$ is to assume that $\Lambda_{Z_{0}}$ equals $\Lambda_{Z}$; i.e., the smoothness of the Gt-f $Z$ under the null hypothesis is the same as the smoothness of the actual realization $Z$. This is approximately true for data showing no or only a few effects (deviations from the null hypothesis), but in most cases this assumption is wrong due to strong activations induced by the paradigm, rendering the smoothness estimate biased. An estimator of the covariance matrix of the partial derivatives of a GRF $Z$ solely on the field $Z$ is

$$
\hat{\lambda}(Z)_{j k}=\frac{\Sigma_{\mathbf{x}}\left(\partial Z(\mathbf{x}) / \partial l_{j}\right)\left(\partial Z(\mathbf{x}) / \partial l_{k}\right)}{\Sigma_{\mathbf{x}} Z(\mathbf{x})^{2}},
$$

which is the ratio of the total sum of the covariances of the partial derivatives divided by the total variance of $Z$ over all positions $\mathbf{x}$.

\section{RESULTS}

We have validated the new smoothness estimator on residuals of simulated one-dimensional functional images and on a fMRI data set. Second, we estimated the smoothness on the statistical images (Gt-f $Z$ ) of the simulated studies to show the bias and higher variance of this estimator. Our finding is that the new estimator based on the residuals is more accurate and more efficient (i.e., has a lower variance) than the estimator based on the statistical image.

Although it was not possible to measure the performance of a smoothness estimator on real data (for which the component fields are not known), we found that estimation of the smoothness of $Z_{0}$ from real data compared well to the expected smoothness induced by the exogenous smoothing prior to analysis.

\section{Validation of New Method on Simulated Data}

One-dimensional data. All validation used simulated one-dimensional GRFs, which was sufficient and computationally efficient, since we assumed that the covariances of $\Lambda_{\epsilon}$ of real three-dimensional observations were zero (Poline et al., 1995).

We performed two sorts of validation. The first was to test, on a set of noise only data, whether the smoothness was correctly estimated under the true null hypothesis. The second data set included some additive signal and we tested whether the smoothness was biased by this underlying signal. Both tests were performed on both temporally independent and temporally autocorrelated data. We used a Gaussian filter kernel to spatially smooth all simulated data sets.

Noise only. We generated series of one-dimensional Gaussian random fields $Y=\mathbf{Y}_{1}, \ldots, \mathbf{Y}_{n}$ by convolving white noise vectors $(8192$ elements, $\sim N(0,1))$ with a Gaussian filter kernel with standard deviation $s$. We then multiplied the convolved white noise field by a variance field (cf. Eq. (5)), where the variance $\sigma_{\mathbf{x}}$ at position $\mathbf{x}$ was distributed with $\sigma_{\mathbf{x}}^{2} \sim N(5,3)$. Although it is unlikely that the chosen distribution simulated the underlying variance map of a real study, it is sufficient to show that the smoothness estimator performs correctly with heterogenous variance. Spatially varying error is a central assumption made in most analyses of functional imaging studies (e.g., in the framework of SPM).

We generated data sets for various different FWHM of the filter kernel used $\left[\mathrm{FWHM}_{1}=2, \mathrm{FWHM}_{2}=3\right.$, $\mathrm{FWHM}_{3}=25$ (voxel)], 104 different degrees of freedom, ranging from 6 to 110, and two values of temporal autocorrelation, where the standard deviations of the Gaussian filter kernels were 0 (independent measurements) and 0.71 scans. The latter value corresponds to a Gaussian kernel with 6-s FWHM. The choice of the different filter widths (2, 3 , and 25 voxels) represents two different situations: (i) an intrinsic smoothness of 2 to 3 voxels induced by an exogeneous lowpass filter which is often the case in real functional studies and a (ii) theoretical case with a very high degree of smoothness (25 voxels FWHM). The latter was used to show that in the limit of high smoothness (i.e., in the absence of discretization errors) the estimates are accurate. For each of these combinations, we generated 32 data sets. We then performed all steps of a SPM analysis (estimating parameter vectors $\hat{\beta}$ for each study, computing a $t$-field $t$ and Gaussianizing $t$ to generate $Z$ ). Finally, the mean and variance of the 32 resulting smoothness estimates of $Z_{0}$ were computed for each parameter combination on the standardized residuals as described above (Eq. (16)), giving us $3 \times 104 \times 2$ (three different FWHM of the spatial filter, 104 different degrees of freedom, two different values of temporal autocorrelation) points in parameter space for our simulated noise only data.

The theoretical value of each $\lambda_{Z_{0}}$ (the variance of the partial derivative of the one-dimension Gaussianized $t$-field under the null hypothesis) can be assessed by

$$
\lambda_{Z_{0}}=\lambda_{v} \cdot \lambda
$$

where

$$
\lambda=\left(\frac{2 \mathrm{FWHM}}{\sqrt{8 \log 2}}\right)^{-1} .
$$



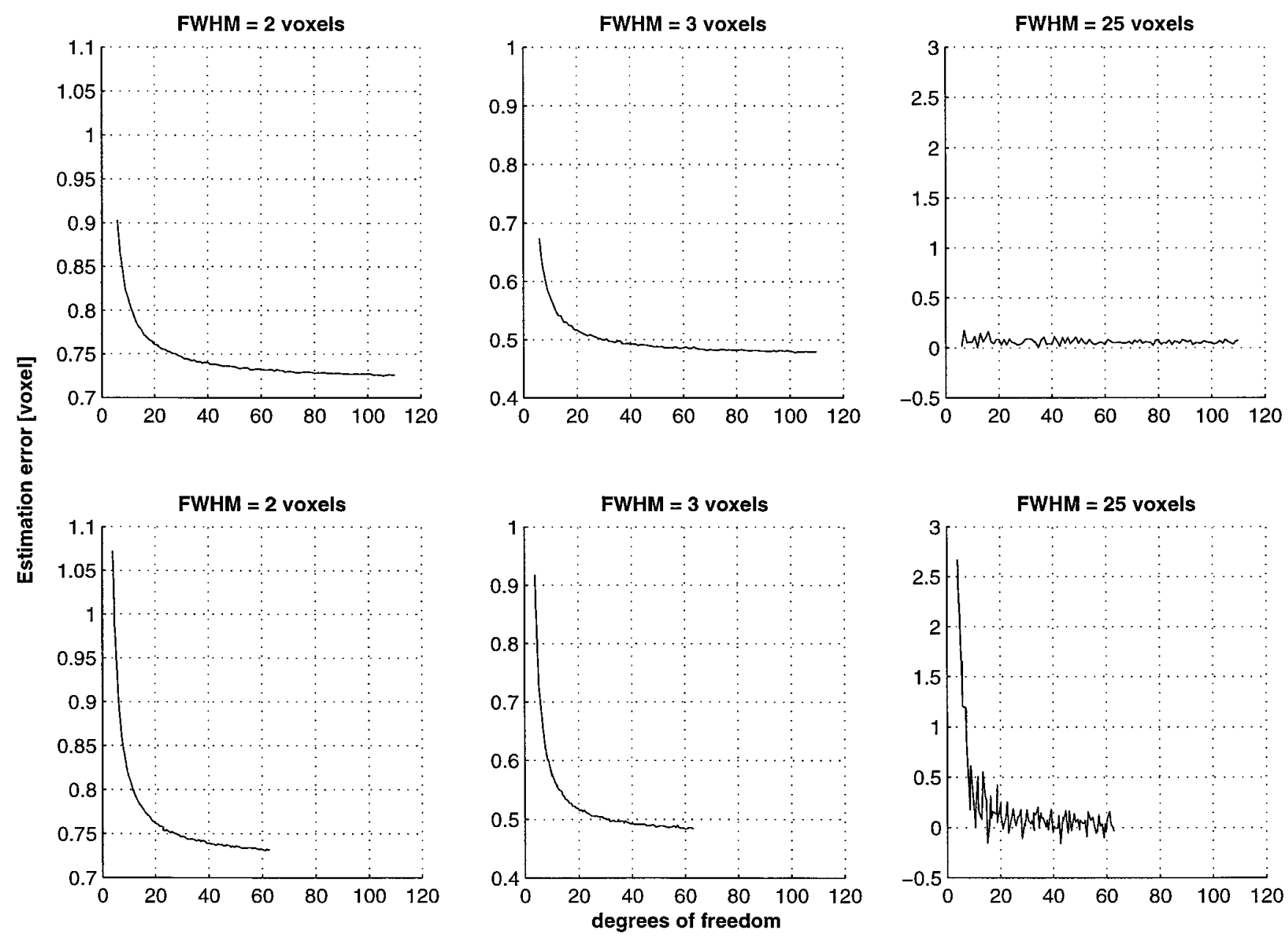

FIG. 3. (Top row) Estimation error for noise data without temporal autocorrelation. (Bottom row) Estimation error for noise data with temporal autocorrelation (Gaussian kernel, $\sigma=0.71$ ). The error is defined as the estimated minus the theoretical FWHM.

In Fig. 3, the errors made by the smoothness estimator are plotted as a function of degrees of freedom for the different smoothing filters $\left[\mathrm{FWHM}_{1}=2, \mathrm{FWHM}_{2}=\right.$ $3, \mathrm{FWHM}_{3}=25$ (voxel)] and two different temporal autocorrelations. The error is defined as the difference between the (known) theoretical and the estimated FWHM of the Gaussian PRF of $Z_{0}$.

The smoothness estimates approximate a biased estimate for high degrees of freedom, where the bias seems to be a function of the FWHM of the filter used to generate the data and the degrees of freedom. The estimates are nearly unbiased for a wide filter kernel and high degrees of freedom. As we will see in the Discussion, these overestimation effects for low degrees of freedom and low FWHM (voxels) are expected and are explained by discretization effects. Moreover, the smoothness estimates on temporally correlated data have higher variance than on uncorrelated data. This seems to be true for all filter sizes, but becomes only obvious for the 25-voxel FWHM, since the variance of the absolute error made by the estimator increases proportionally with the width of the underlying filter kernel.

Linear additive signal. In contrast to the noise only validation, a second study based on simulated data was performed which included an additive activation effect. Data were generated as before, but this time a signal (a constant value of 0.3 ) prior to the convolution with the PRF was added to 1000 (of 8192) contiguous voxels in an alternating on-off pattern over time. The signal was modeled by two linear basis functions so that this experiment simulated a simple activation study with two conditions.

We used the same parameters as in the noise only study, giving us $3 \times 104 \times 2$ (three different FWHM of the spatial filter, 104 different degrees of freedom, two different values of temporal autocorrelation) parameter sets. We generated 32 data sets for each parameter combination and estimated its smoothness on the standardized residuals.

The results are nearly indistinguishable from the 


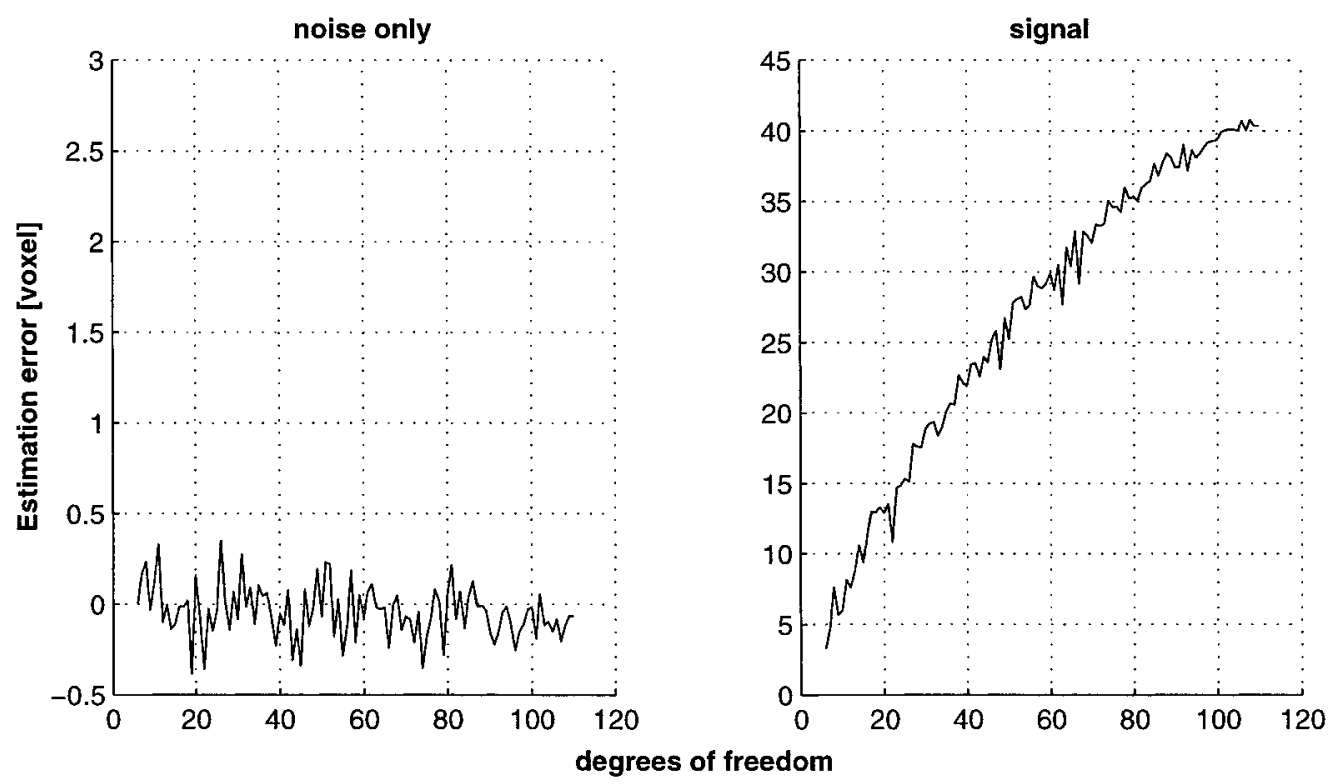

FIG. 4. Estimation error of estimator based on statistical image. (Left) Error on noise data; (Right) error on data with additive signal. The error is defined as the estimated minus the theoretical FWHM.

noise only study (Fig. 3) and therefore the corresponding curves are not displayed.

\section{Smoothness Estimation Based on the Statistical Image}

As described above, the smoothness of the statistical image $Z_{0}$ under the null hypothesis can be assessed directly on the statistical field $Z$. To show the bias due to a potential signal and the higher variance of such an estimator, we estimated the smoothness on the same simulated studies as used in the previous section, but based on Eq. (19). We applied the estimator only to simulated studies, which were convolved with a Gaussian filter kernel with a FWHM of 25 voxels to exclude confounding effects due to coarse filter sampling (see Discussion). Furthermore, we assumed temporally independent data. Results are very similar for autocorrelated data (data not shown).

Figure 4 shows the estimates based on a noise only and a signal data set (25-voxel FWHM). We used 32 studies to compute the mean of 32 smoothness estimates for each data point as a function of degrees of freedom.

The differences between the two smoothness estimators are evident by comparison of Fig. 3 with Fig. 4. First, the method based on the statistical image clearly overestimates the underlying smoothness, if the statistical image contains effects due to an underlying signal, and, second, the estimates have a rather high variance as can be seen by comparison with the two right-most graphs in Fig. 3.

\section{Application of the Smoothness Estimator to an fMRI Study}

To illustrate the performance of the new smoothness estimator on real data, we applied the estimator given by Eq. (14) to a single subject fMRI activation study. We used SPM96, since this implementation contained the smoothness estimator based on the residuals.

Data were acquired with a 2-T MRI (Magnetom Vision, Siemens, Erlangen, Germany) whole-body MRI equipped with a head volume coil. Contiguous multislice $\mathrm{T} 2 *$-weighted images $(\mathrm{TE}=40 \mathrm{~ms}, 90 \mathrm{~ms} / \mathrm{image}$, $64 \times 64$ pixels, voxel size $3 \times 3 \times 3 \mathrm{~mm}^{3}$ ) were obtained with echo-planar imaging (EPI) using an axial slice orientation. The volume covered the whole brain $(14.4 \mathrm{~cm})$. The effective repetition time was $4.0 \mathrm{~s}$ per volume.

The paradigm used was a simple finger tapping condition alternated with rest.

Prior to statistical analysis we spatially smoothed the data with an isotropic Gaussian filter with a FWHM of three voxels in each direction. Figure 5 contains the SPM96 statistics output for this data set. As it can be seen, the smoothness based on the residuals is estimated as $(3.7,3.6,3.4)$ FHWM (voxel) in $x$-, $y$-, and $z$-direction, which is an overestimation of the theoretically expected smoothness of three-voxel FWHM. However, the major part of this overestimation is most likely due to filter discretization effects (see Discussion) and physiologically mediated effects.

\section{DISCUSSION}

In this paper we have described a new smoothness estimator for discrete Gaussian random fields based on the residuals of a general linear model.

It is important to note that the new smoothness estimator combined with the possibility of relating smoothness estimates of subsequent processing stages 


\section{$\operatorname{SPM}\{Z\}$}
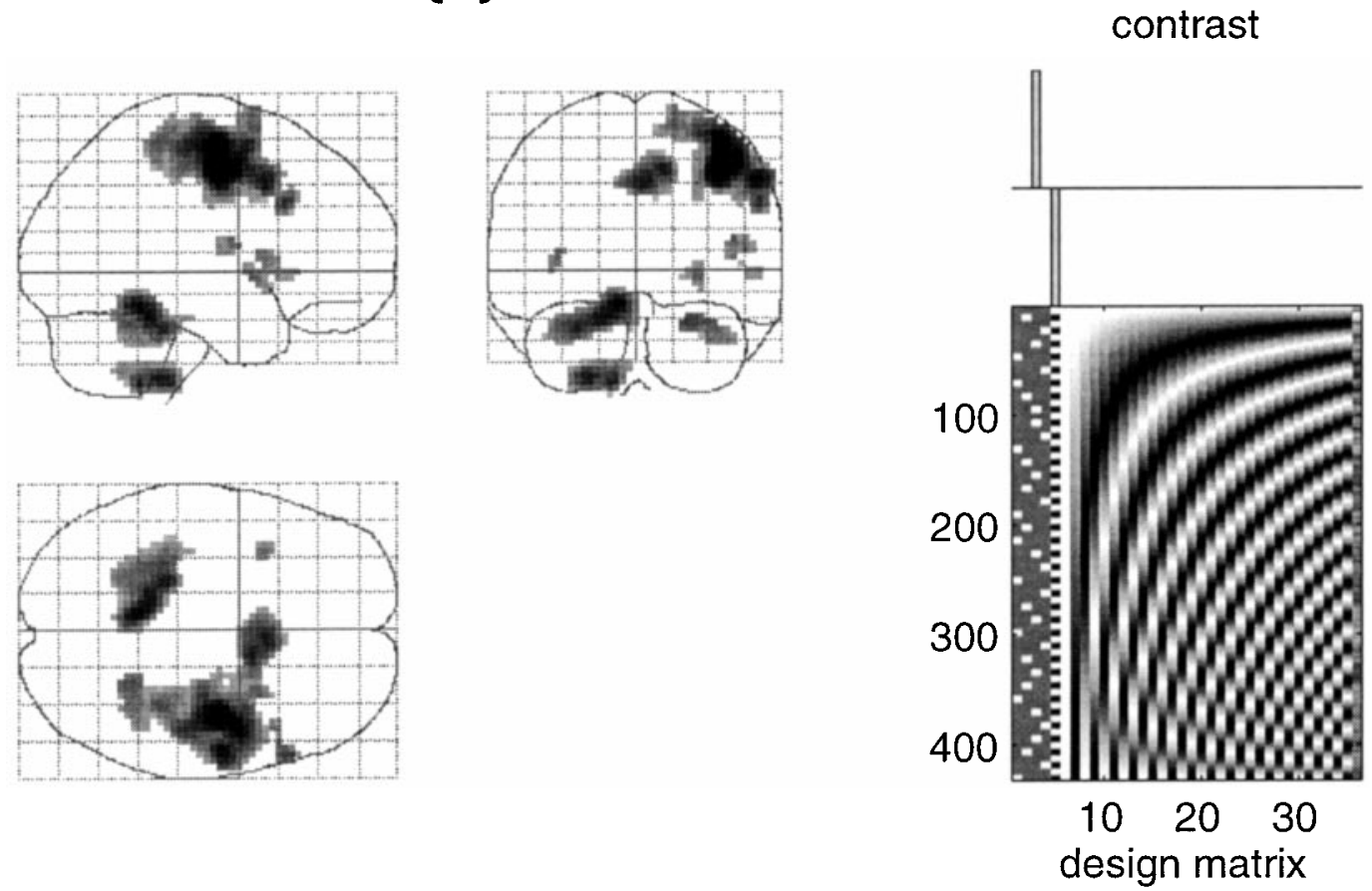

\section{$P$ values \& statistics:}

\begin{tabular}{|c|c|c|c|c|c|c|c|c|}
\hline \multirow{2}{*}{$\frac{\text { set-level }\{c\}}{0.985(10)}$} & cluster-level $\{k, Z\}$ & \multicolumn{2}{|c|}{ voxel-level $\{Z\}$} & \multicolumn{2}{|c|}{ uncorrected $\mathrm{k} \& \mathrm{Z}$} & \multicolumn{3}{|c|}{$\mathrm{x}, \mathrm{y}, \mathrm{z}\{\mathrm{mm}\}$} \\
\hline & $0.000(1162,8.13)$ & 0.000 & (8.13) & 0.000 & 0.000 & 39 & -9 & 48 \\
\hline & & 0.000 & $(8.08)$ & & 0.000 & 39 & -9 & 57 \\
\hline & & 0.000 & $(7.37)$ & & 0.000 & 54 & -9 & 42 \\
\hline & $0.000(405,7.50)$ & 0.000 & (7.50) & 0.000 & 0.000 & -9 & -45 & -15 \\
\hline & & 0.000 & (6.32) & & 0.000 & -27 & -36 & -27 \\
\hline & $0.001 \quad(189,6.98)$ & 0.000 & (6.98) & 0.000 & 0.000 & -21 & -36 & -48 \\
\hline & & 0.000 & $(6.17)$ & & 0.000 & -12 & -45 & $5-51$ \\
\hline & $0.002(223,6.70)$ & 0.000 & $(6.70)$ & 0.000 & 0.000 & 3 & 12 & 42 \\
\hline & & 0.000 & $(6.48)$ & & 0.000 & 12 & 6 & 48 \\
\hline & $0.033 \quad(34,5.41)$ & 0.002 & $(5.41)$ & 0.072 & 0.000 & 57 & 21 & 33 \\
\hline & $0.088 \quad(93,4.98)$ & 0.018 & $(4.98)$ & 0.006 & 0.000 & 24 & -48 & -24 \\
\hline & & 1.000 & $(2.60)$ & & 0.005 & 36 & -30 & -30 \\
\hline & $0.581 \quad(27,4.14)$ & 0.427 & $(4.14)$ & 0.105 & 0.000 & 27 & & -3 \\
\hline & $0.732(17,4.00)$ & 0.607 & $(4.00)$ & 0.190 & 0.000 & -36 & 12 & 6 \\
\hline & $0.759 \quad(23,3.97)$ & 0.642 & (3.97) & 0.131 & 0.000 & 45 & -3 & 15 \\
\hline & & 1.000 & (3.18) & & 0.001 & 51 & -9 & 12 \\
\hline & $0.990 \quad(19,3.55)$ & 0.982 & (3.55) & 0.168 & 0.000 & 45 & 15 & 6 \\
\hline & & 1.000 & (2.93) & & 0.002 & 54 & 18 & 0 \\
\hline
\end{tabular}

Height threshold $\{u\}=2.33, p=0.010$

Extent threshold $\{k\}=11$ voxels, $p=0.289$

Expected voxels per cluster, $E\{n\}=10.6$

Expected number of clusters, $E\{m\}=18.0$
Volume $\{S\}=65809$ voxels or 1471.5 Resels

Degrees of freedom due to error $=225.1$

Smoothness FWHM $\{\mathrm{mm}\}=11.010 .810 .1$

$\{$ voxels $\}=3.73 .63 .4$

FIG. 5. Output of SPM96 statistics section. 
of SPM to each other is a powerful tool to estimate smoothness of other statistic fields under the null hypothesis. It is, e.g., possible to relate the smoothness of the residual components to the smoothness of the $F$-field such that $P$ values for peak height in the $F$-field can be estimated (Worsley, 1994).

We have shown empirically for simulated data that the smoothness estimator will be unbiased if (1) the degrees of freedom of the model are sufficiently high $(>20)$ and (2) the discrete filter kernel used for spatial filtering is a fine mesh representation of the underlying continuous filter. This is true for both temporally independent and autocorrelated data. As expected, the smoothness estimated is invariant of a linear additive signal modeled as a temporal basis function in the design matrix of the specified general linear model. However, in real studies, any signal components not modeled as basis functions will bias the smoothness estimate.

Some overestimation can be observed in Fig. 4, which shows the estimates based on the statistical images. The estimates are clearly an overestimation of the known underlying smoothness, if there is a smooth signal component present in the statistical image. Moreover, the estimates have a higher variance. To explain the massive overestimation, we estimate the effect of basing the estimate on the statistical image $Z$ :

If some signal is present in $Z$, we can split the total variance into two components,

$$
\int E\left(Z^{2}\right)=\int \operatorname{Var}(Z)+\int E(Z)^{2},
$$

and the derivative is

$$
\int E\left(\dot{Z}^{2}\right)=\int \operatorname{Var}(\dot{Z})+\int E(\dot{Z})^{2}
$$

so that the expectation of $\hat{\Lambda}_{Z}$ is given by

$$
E\left(\hat{\Lambda}_{Z}\right)=\frac{\int E\left(\dot{Z}^{2}\right)}{\int E\left(Z^{2}\right)}=\frac{\Lambda_{N}+\sigma_{S}^{2} \Lambda_{S}}{1+\sigma_{S}^{2}},
$$

where $\Lambda_{N}$ is the underlying component of $\Lambda_{Z}$ due to noise, $\Lambda_{S}$ is due to the signal not removed from $Z$, and $\sigma_{S}^{2}$ is the average variance of the signal. Quite clearly, if the smoothness of the noise is the same as the smoothness of the signal $\left(\Lambda_{N}=\Lambda_{S}\right)$, the estimated $\Lambda_{Z}$ will be the true $\Lambda_{N}$. However, if the signal component is smoother than the noise, $\Lambda_{Z}$ will be greater than $\Lambda_{N}$ and the smoothness of the statistical image under the null hypothesis will be overestimated. This behavior of the estimator based on the statistical image should be expected for any functional brain images, since the underlying physiological signal seems always to be smoother than the noise measured. Given the unlikely case that the signal component in functional brain images is rougher than the underlying noise, then Eq. (23) makes it clear that the estimated $\Lambda_{Z}$ would be greater than $\Lambda_{N}$ and smoothness would be underestimated.

The higher variance of the estimator based on the statistical image compared to the estimator based on the residuals is due to the smaller number of samples present in the statistical image $Z$ compared to the overall number of voxels over all residual components. There is only one statistical image, but many more observations and the same number of residual components such that the estimator has a lower variance.

Returning to the estimator based on the standardized residuals, we need some more theoretical insight to correctly interpret the results presented in Fig. 3. In the following, we show that the quantization error induced by the discretization of a continuous filter kernel influences the smoothness estimation performed on a stationary field convolved with this discrete filter.

Generally, the variance of the partial derivative of a smooth stationary field is given by

$$
\operatorname{Var}(\dot{Z})=-\sigma^{2} \ddot{\rho}(0)
$$

where $\rho$ is the ACF of the stationary field $Z$ (Cox and Miller, 1996). This is the analytical form to compute the variance of the partial derivative provided that the second derivative of the ACF at zero is known. Assume that we undersample a known continuous Gaussian filter kernel and use this discretized PRF for convolution of a discrete stationary Gaussian random field as we did it in our simulated data studies. The sampled approximation to a continuous Gaussian envelope has lower slopes at the points of inflection which in turn results in lower maxima of the first derivative such that the minimum of the second derivative at zero is lower than the equivalent value of the continuous Gaussian envelope. Note that different scaling factors of the continuous filter and its discrete counterpart do not play a role here, since these factors cancel out because of the $t$-field equation (Eq. (9)) and the voxelwise variance estimation (Eq. (8)). As a consequence, $V(\dot{Z})$ is lower than expected and the smoothness $|\Lambda|^{-1 / 2}$ is overestimated in the sense that we wish to estimate the smoothness induced by a continuous Gaussian kernel whose sampled version we used to smooth the discrete stationary field. This smoothness overestimation due to discretization effects is a decreasing function of the FWHM and the degrees of freedom. This can be observed in Fig. 3. The overestimation is at maximum for very low degrees of freedom and for a FWHM as small as two voxels. The approximate nature of the function $f(d f)=(d f-2 / d f-1)$ renders this effect less severe for higher degrees of freedom $(d f>20)$, although a small bias, depending on the underlying FWHM, can be 
observed in all estimates. For very high FWHM (e.g., 25 voxels) this overestimation is negligible.

Another source of error due to the discreteness of the functional data is the estimation of $P$ values based on the statistical image. Any undersampled lattice representation of a continuous Gaussian random field has lower maxima than the unobservable continuous version so that resulting $P$ values are lower for the discrete case.

\section{CONCLUSION}

We have demonstrated the accuracy of an estimation method for the smoothness of discrete approximations to continuous Gaussian random fields. Using simulated data we have shown that errors in smoothness estimation depend (in an expected way) on the degrees of freedom and on the size of the spatial filter kernel applied prior to statistical analysis.

\section{REFERENCES}

Adler, R. 1981. The Geometry of Random Fields. Wiley, New York. Cox, D., and Miller, H. 1996. The Theory of Stochastic Processes. Chapman and Hall, London.
Frackowiak, R., Friston, K., Frith, C. D., Dolan, R., and Mazziotta, J. C. 1997. Human Brain Function. Academic Press, San Diego.

Friston, K. J., Frith, C. D., Liddle, P., and Frackowiak, R. S. 1991. Comparing functional (PET) images: The assessment of significant change. J. Cereb. Blood Flow Metab. 11:690-699.

Friston, K. J., Poline, J.-B., Holmes, A. P., Price, C. J., and Frith, C. D. 1996. Detecting activations in PET and fMRI: Levels of inference and power. NeuroImage 4:223-235.

Friston, K. J., Worsley, K. J., Frackowiak, R. S., Mazziotta, J., and Evans, A. C. 1994. Assessing the significance of focal activations using their spatial extent. J. Hum. Brain Map. 1:214-220.

Holmes, A. P. 1994. Statistical Issues in Functional Neuroimaging. Ph.D. thesis, University of Glasgow.

Poline, J.-B., Worsley, K. J., Holmes, A. P., Frackowiak, R. S., and Friston, K. J. 1995. Estimating smoothness in statistical parametric maps: Variability of p-values. J. Comput. Assist. Tomogr. 19(5):788-796.

Worsley, K., and Friston, K. 1995. Analysis of fMRI time-series revisited-Again. NeuroImage 2:173-181.

Worsley, K. J. 1994. Local maxima and the expected euler characteristic of excursion sets of $\chi^{2}, \mathrm{~F}$ and t-fields. Adv. Appl. Probability 26:13-42.

Worsley, K. J. 1996. An Unbiased Estimator for the Roughness of a Multivariate Gaussian Random Field. Technical report, Department of Mathematics and Statistics, McGill University.

Worsley, K. J., Evans, A. C., Marrett, S., and Neelin, P. 1992. A three-dimensional statistical analysis for CBF activation studies in human brain. J. Cereb. Blood Flow Metab. 12:900-918. 